\title{
Repenser le processus de raisonnement en soudage- montage : interdépendance entre professionnalisation, employabilité et partenariat
}

\author{
Auteures \\ Marie Alexandre, Ph. D., Professeure en psychopédagogie de \\ l'enseignement professionnel, Université du Québec à Rimouski, \\ Canada, \\ Marie Alexandre@uqar.ca
}

Dominique Amyot, Agente de recherche

Université du Québec à Rimouski, Canada,

Dominique Amyot@uqar.ca 


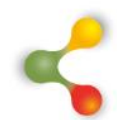

\section{REVUE HYBRIDE DE L'ÉDUCATION}

\section{Résumé}

Cet article traduit une préoccupation quant à la complexification des situations de travail du XXIe siècle. Prenant appui sur un modèle de développement de compétences durables inscrit en didactique professionnelle, dix personnes expérimentées en soudage-montage ont participé à une recherche qualitative sur le processus de travail. Une démarche d'analyse de contenu a permis de décrire les paliers décisionnels et opérationnels des activités clés et des paramètres de sécurité, de métier et de conformité associés aux actions du processus de raisonnement. Les résultats illustrent des manifestations de l'intelligence professionnelle en soudage-montage et l'interdépendance de la professionnalisation, de l'employabilité et du partenariat.

Mots-clés: compétences; formation professionnelle; didactique professionnelle; processus de raisonnement de métier; savoir de métier 


\section{8}

\section{REVUE HYBRIDE DE L'ÉDUCATION}

\section{Problématique}

L'arrimage entre l'enseignement professionnel et le marché du travail inclut la formation et le développement des compétences (Bureau international du travail, 2011). Or le constat est préoccupant. L'Organisation internationale du travail (2014) rapporte un décalage entre les compétences acquises en formation et la nature du travail disponible. Le Conseil consultatif en matière de croissance économique et le Forum des ministres canadiens du marché du travail font valoir l'urgence d'adopter de nouvelles approches afin de combler les écarts de compétences et d'appuyer la formation continue tout au long de la vie professionnelle des Canadiens (gouvernement du Canada, 2017). Au Québec, alors que 1,4 million d'emplois seront à pourvoir au cours des prochaines années, les nouvelles exigences quant à la formation des travailleurs (Perform, 2012, 2017), le retard concernant la qualification et l'acquisition des compétences, ainsi que la pénurie de la main-d'œuvre menacent la croissance économique du Québec (gouvernement du Québec, 2013).

Par ailleurs, l'examen des dimensions de la formation et du travail indique des organisations, des environnements, des groupes sociaux et des individus porteurs de logiques différentes (Wittorski, 2016). La réduction de l'inadéquation entre la formation et l'emploi (skill mismatch) suppose un travail de mise en correspondance entre deux champs; celui de la formation et celui de l'activité de production qui s'y rapporte (Doray, Simoneau et Solar-Pelletier, 2017 ; McGuinness, Pouliakas et Redmond, 2017).

Dans ce contexte de diversification et de complexification, les acteurs (enseignants, formateurs et travailleurs) de la formation professionnelle doivent s'adapter aux nouvelles exigences tout en anticipant les changements. Plus spécifiquement, dans le cas du secteur de la fabrication métallique du Québec, le déficit anticipé annuel de la maind'œuvre en soudage-montage serait de 2300 personnes alors que seulement 900 obtiendraient leur diplôme d'études professionnelles (Perform, 2015). Non seulement le nombre de diplômés continue de décroître, mais aussi les travailleurs ne possédant pas de diplôme d'études professionnelles stagnent dans leur développement professionnel au sein de l'entreprise (Perform, 2014). En effet, la modification des processus de production et des méthodes de travail implique une hausse significative des exigences envers la main-d'œuvre (Champy-Remoussenard, 2008, Trottier 2005). En soudage-montage, on constate des exigences accrues concernant les compétences en assemblage (ou montage), la maîtrise de plusieurs techniques de soudage (FCAW, GMAW, SMAW, GTAW, etc.), la connaissance des propriétés des métaux et les aptitudes requises en lecture de plans (Emploi-Québec, 2018 ; gouvernement du Canada, 2018 ; Perform, 2015). 


\section{6}

\section{REVUE HYBRIDE DE L'ÉDUCATION}

Considérant que ce sont les acteurs du milieu qui construisent les ponts entre la formation et l'emploi et donnent tout son sens à cette adéquation, cet article rend compte des résultats d'une étude ${ }^{1}$ menée en soudage-montage auprès d'enseignants, de formateurs en milieu de travail et de travailleurs sur l'agir professionnel. Prenant appui sur les travaux de la didactique professionnelle, le processus de raisonnement de métier est défini au regard de l'analyse de la dimension cognitive de l'activité (Alexandre, Thériault et Daigle, 2016 ; Pastré, 2002) et de la mobilisation des processus cognitifs en situation de travail dans le développement des compétences.

\subsection{Processus de raisonnement de métier}

Pour la didactique professionnelle, l'identification de la dimension cognitive de l'action est un enjeu de taille pour les experts, les moins experts, et pour ceux qui sont en cours d'apprentissage. En effet, l'analyse conduit non seulement à l'identification des connaissances et des capacités utiles à la situation, aux modes de raisonnement en action, mais en plus elle distingue des niveaux de compétence. La didactique professionnelle propose une série de cadres théoriques pour examiner et interpréter l'action et son organisation cognitive (Fournier, Lambert et Marion-Vernoux, 2017; Pastré, 2002 ; Pastré, Mayen et Vergnaud, 2006 ; Rogalski, 2004). L'analyse du sens de l'action permet de saisir les points critiques qui composent le parcours de développement des compétences.

Parmi les travaux relatifs au processus de travail, des études ont montré " une forme d'intelligence des situations » définie comme étant un processus décisionnel, caractérisé par une mobilisation de l'ensemble des ressources individuelles, une forme de diagnostic et une organisation de stratégies d'action (Alexandre, Thériault et Daigle, 2016). Les activités perceptives, les prises d'information, l'identification de l'état et de l'évolution des situations d'action ainsi qu'une suite complexe de raisonnements, de choix, de contrôles et d'ajustements constituent les registres cognitifs qui servent de base à la conceptualisation des situations professionnelles. Les savoirs produits et détenus par les praticiens à partir d'activités réelles du domaine assurent la cohésion de l'action, de la pensée et du savoir (Holgado et Mayen, 2017; Leplay, 2006). Cependant, les connaissances scientifiques actuelles dans ce domaine laissent encore plusieurs éléments sans réponse.

\footnotetext{
${ }^{1}$ Cette recherche a été réalisée avec le soutien du Conseil régional des partenaires du marché du travail (Bas-Saint-Laurent), Emploi-Québec Bas-Saint-Laurent, le ministère de l'Éducation, de l'Enseignement supérieur et de la Recherche, le Cégep de Rivière-duLoup, le Comité sectoriel de la main-d'œuvre de la fabrication métallique du Québec et I'Université du Québec à Rimouski.
} 


\section{$\&$}

\section{REVUE HYBRIDE DE L'ÉDUCATION}

Les processus cognitifs influencent le développement des personnes à moyen et à long terme. Ils sont au cœur de " l'évolution des compétences et de leurs relations au cours de l'expérience " (Pastré, Mayen et Vergnaud, 2006, p.154). La prise en compte des dimensions de la situation de travail, des actions posées et des résultats attendus témoigne de la pensée réflexive qui sous-tend l'agir professionnel. Ainsi le processus de raisonnement de métier correspond-il à une "délibération » en temps réel constitué de paliers décisionnels et opérationnels (Alexandre, Daigle et Amyot, 2019). Ce réseau complexe des activités clés et des actions qui en découlent propose une formalisation explicite de la pensée de métier exercée en situation de travail (Alexandre, Thériault et Daigle, 2016).

Toutefois, la réduction du décalage entre les compétences acquises en formation et les exigences du milieu de travail requiert une meilleure description de l'agir professionnel, en portant une attention particulière aux interactions entre l'individu, son activité et son environnement. Ainsi les concepts porteurs de l'interdépendance des logiques inhérentes à chacun des environnements (Wittorski, 2016) jettentils les bases d'une écologie de l'apprentissage du travail en vue d'une meilleure adéquation entre la formation et l'emploi ?

\subsection{Adéquation entre la formation et l'emploi}

Une revue récente d'écrits scientifiques (Bernier, Michaud et Poulet, 2017) montre que les recherches en adéquation formation-emploi sont généralement abordées sous des angles distincts. À l'heure actuelle, les chercheurs qui se sont penchés sur l'analyse du travail ont principalement examiné de façon isolée les concepts de professionnalisation, de partenariat et d'employabilité auprès de travailleurs ou de formateurs. De plus, les devis utilisés par ces études ont peu articulé les liens entre éducation et emploi dans une visée systémique de l'apprentissage du travail. En outre, une recension des écrits scientifiques sur les processus cognitifs (Nendaz, Charlin, Leblanc et Bordage, 2005 ; Wanlin, 2009) dans différents champs scientifiques tels que la santé (raisonnement clinique) et les sciences de l'éducation (teacher thinking) a montré que les théories des décisions et celles du traitement de l'information étaient principalement étudiées dans le cadre de formations universitaires, se limitant souvent au seul point de vue du praticien sans l'apport des autres acteurs du milieu du travail, de l'enseignement ou de la formation hors du contexte scolaire. Il est important de combler ces lacunes compte tenu des implications sur la maîtrise des situations de travail en formation professionnelle.

Le cadre conceptuel de l'étude, présenté dans la section suivante, est fondé sur les relations plurielles et multidimensionnelles entre l'éducation et le marché du travail. L'unité fonctionnelle formation-travailapprentissage proposée par Holgado et Balleux (2015) permet une 


\section{8}

\section{REVUE HYBRIDE DE L'ÉDUCATION}

meilleure compréhension des milieux dans lesquels se forment les professionnels tout en adoptant une visée systémique de l'apprentissage du travail. Dans une perspective intégratrice, la notion de processus de raisonnement de métier prend en compte les interactions entre les trois axes privilégiant les concepts de professionnalisation (axe apprentissageformation), d'employabilité (axe travail-apprentissage) et de partenariat (axe formation-travail).

\section{Cadre conceptuel}

Les praticiens conjuguent la pensée, le savoir et l'action dans la production du savoir professionnel (Leplay, 2006). La modélisation du développement des compétences durables en formation professionnelle et technique (Alexandre, 2018), présentée à la figure 1 ci-dessous, inscrit dans un système interdépendant trois concepts porteurs de l'apprentissage du travail: la professionnalisation, l'employabilité et le partenariat. Au centre du système, le processus de raisonnement de métier articule les liens entre les champs de la formation et de l'emploi, et permet de retracer les interactions entre l'individu, son activité et son environnement.

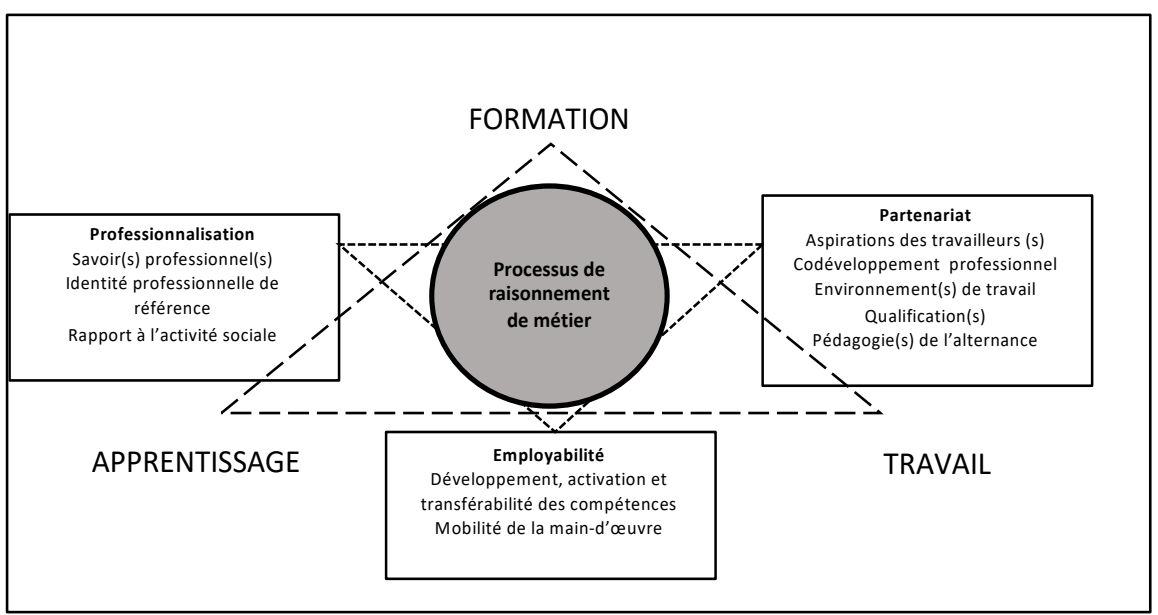

Figure 1 : Modélisation du développement des compétences durables en formation professionnelle et technique (Alexandre, 2018)

\subsection{Axe apprentissage-formation : Professionnalisation}

Selon Wittorski (2016), l'étude de la professionnalisation est interdépendante des enjeux liés à la transformation du travail et à l'ouverture à des environnements dépassant le cadre de la formation. La professionnalisation se définit selon un espace de production de biens et de services organisé autour d'une intention de développement de compétences qui permet à l'individu de se préparer à l'exercice d'une activité professionnelle donnée (Barbier, 2012; Wittorski 2016). Plus 


\section{$\&$}

\section{REVUE HYBRIDE DE L'ÉDUCATION}

particulièrement, Champy-Remoussenard (2008) souligne que la professionnalisation est un indicateur de la relation entre l'éducation, la formation et l'emploi. L'axe apprentissage-formation inclut l'acquisition et la mise en œuvre des savoirs professionnels (Jobert, 2002), la construction d'une identité professionnelle de référence (Fray et Picouleau, 2010; Perrenoud, 2004) et l'inscription d'un rapport spécifique à l'activité sociale (Champy-Remoussenard, 2008).

\subsection{Axe travail-apprentissage : Employabilité}

L'axe travail-apprentissage prend en compte l'activation et la transférabilité des compétences liées à l'employabilité (Bricler, 2009; Pastré, Mayen et Vergnaud, 2006). Pour Santelmann (2019), l'employabilité est porteuse d'évolution professionnelle. Bros, et al. (2019) soulignent l'importance des compétences transversales et transférables dans le soutien de l'employabilité. En effet, les compétences transversales constituent un levier pour le développement des compétences disciplinaires et un élément important de distinction entre les experts et les novices (gouvernement du Québec, 2019). Selon Dujardin (2013), pour être durable, une compétence doit être périodiquement activée et transférable.

Toutefois, Bros, et al. (2019) soulignent le risque de mobiliser la notion de compétence transversale dans la rédaction de référentiels. Selon ces derniers, la notion de compétence «saisie hors de son ancrage dans le travail réel » (p.157) serait dénuée de sens, même si le gouvernement du Québec (2019) admet que les compétences transversales se construisent et prennent racine dans des contextes d'apprentissage spécifiques et disciplinaires. Le programme de formation de l'école québécoise décrit des compétences transversales d'ordre intellectuel, méthodologique, personnel, social et communicationnel (gouvernement du Québec, 2019).

Pour sa part, Bricler (2009) insiste sur la mobilité de la maind'œuvre et la capacité d'appliquer et de maîtriser une compétence dans des situations nouvelles et possiblement plus complexes. En outre, l'employabilité durable sécurise les parcours professionnels face à l'évolution rapide des métiers, des techniques et de l'organisation du travail. Elle correspond à la capacité de l'individu, à tout moment de sa vie professionnelle, de conserver, de (re)trouver un emploi dans des délais raisonnables (Bricler, 2009).

Dans une recherche menée auprès de 107 employés d'industries en Malaisie, Rasul et ses collaborateurs (2013) font valoir les compétences d'employabilité suivantes : la sécurité au travail, l'intégrité, le service à la clientèle, la résolution de problèmes, la créativité et le leadership. De même, le Conference Board du Canada propose, en 2019, un profil d'employabilité pour la création d'une main-d'œuvre de qualité pour aujourd'hui et demain. II est intéressant de constater qu'au-delà des 


\section{$\&$}

\section{REVUE HYBRIDE DE L'ÉDUCATION}

compétences académiques se retrouvent les qualités personnelles et l'esprit d'équipe. En ce sens, l'employabilité interactive sous-entend la mobilisation d'aptitudes personnelles et de capacités d'adaptation à l'environnement de travail.

Une analyse des compétences présentes dans les référentiels canadiens et américains ${ }^{2}$ a permis des rapprochements avec les travaux de deux chercheurs néerlandais. En 2012, Voogt et Pareja Roblin ont mené une méta-analyse sur 32 documents relatifs à huit grands référentiels nationaux et internationaux ${ }^{3}$ ayant comme objectif la promotion des compétences à enseigner au XXIe siècle. Ces chercheurs rapportent que les compétences relatives aux technologies de l'information et de la communication (TICS) occupent une place centrale autant comme outil de soutien et d'évaluation que de justification pour les nouvelles compétences. Les résultats montrent que la collaboration, la communication, la littératie numérique et les compétences sociales et culturelles incluant l'agir citoyen font consensus. De plus, la créativité, la pensée critique, la productivité et la résolution de problèmes sont largement partagées (Voogt et Pareja Roblin, 2012).

\subsection{Axe formation-travail : Partenariat}

L'adéquation entre la formation et l'emploi englobe diverses dimensions qui tiennent compte des relations plurielles et multidimensionnelles (théoriques, méthodologiques et pratiques) entre l'éducation et le marché du travail (Bernier, Michaud, Poulet, 2017; Simoneau, 2017). Le partenariat correspond à l'ensemble des liens établis entre des organisations sur les différents enjeux d'adéquation entre la formation et le travail. Dignard (2017) souligne les diverses finalités des recherches menées entre la formation et l'emploi, notamment l'employabilité, le développement des compétences, l'intégration en emploi, la pénurie actuelle ou appréhendée de la main-d'œuvre. Le tableau 1 suivant illustre les repères de cette adéquation.

\footnotetext{
2 «Apprentissage au $21^{e}$ siècle (Action Canada, 2013), Compétences du XXle siècle (Canadiens pour l'apprentissage et l'innovation au XXI siècle, 2012) et 21st Century Skills : Learning for Life in Our Times " (Trilling et Fadel, 2009)

${ }_{3}$ Partnership for 21st century skills (P21) (États-Unis); EnGauge (Metiri group and the Learning Point Associates); Assessment and Teaching of 21st Century Skills (ATCS), (Cisco, Intel et Microsoft) ; National Educational Technology Standards (NETS) (International Society for Technology in Education (ISTE)) ; Technological Literacy Framework for the 2012 National Assessment of Educational Progress (NAEP) (WestEd pour National Assessment Governing Board, États-Unis ); 21st century skills and competences for new millennium learners (OCDE) ; Key competences for lifelong learning (OCDE) ; ICT competency framework for teachers, (UNESCO).
} 


\section{REVUE HYBRIDE DE L'ÉDUCATION}

Tableau 1 : Repères de l'adéquation entre la formation et l'emploi (Dignard, 2017)

\begin{tabular}{|c|c|c|}
\hline Niveaux & $\begin{array}{l}\text { Macro : l'adéquation est recherchée en fonction } \\
\text { d'indicateurs économiques ou sociaux, de statistiques } \\
\text { sur la population, de décisions ou d'orientations } \\
\text { politiques. }\end{array}$ & $\begin{array}{l}\text { État, regroupements } \\
\text { d'employeurs, centrale } \\
\text { syndicale }\end{array}$ \\
\hline & $\begin{array}{l}\text { Méso : l'adéquation est recherchée en fonction des } \\
\text { besoins des secteurs de l'industrie, des professions et } \\
\text { des régions. }\end{array}$ & $\begin{array}{l}\text { Région, comités } \\
\text { sectoriels, ordres } \\
\text { professionnels }\end{array}$ \\
\hline & $\begin{array}{l}\text { Micro : l'adéquation est recherchée en fonction des } \\
\text { besoins particuliers d'une entreprise ou d'une personne. }\end{array}$ & $\begin{array}{l}\text { Entreprise, unités de } \\
\text { production, individus }\end{array}$ \\
\hline Types & \multicolumn{2}{|c|}{$\begin{array}{l}\text { Quantitative : l'adéquation vise une correspondance entre des quantités de } \\
\text { personnes formées et d'emplois disponibles. } \\
\text { Qualitative : l'adéquation vise une correspondance entre le contenu d'une } \\
\text { formation et les activités de travail qui s'y rapportent. }\end{array}$} \\
\hline Mesures & \multicolumn{2}{|c|}{$\begin{array}{l}\text { Statistique : l'adéquation se fonde sur une analyse de données sur la formation et } \\
\text { l'emploi : portrait du nombre de personnes formées en regard du nombre de postes } \\
\text { disponibles ou occupés. } \\
\text { Normative : l'adéquation se fonde sur l'évaluation des contenus de formation en } \\
\text { fonction d'une norme : compétences liées à une activité, référentiel d'un diplôme. } \\
\text { Subjective : l'adéquation se fonde sur le jugement d'une organisation ou d'une } \\
\text { personne au sujet de la correspondance entre une formation et l'emploi auquel elle } \\
\text { se rapporte. }\end{array}$} \\
\hline
\end{tabular}

Billett (2004) met de l'avant le statut des lieux de travail comme espaces d'apprentissage qui recèlent des qualités pédagogiques et permettent des expériences centrales pour la continuité de la pratique professionnelle. La qualité des expériences (activités et interactions) autant pour les établissements d'enseignement que pour les lieux de travail détermine la richesse potentielle des résultats de l'apprentissage par le travail. Selon Geay (2007), l'apprentissage en alternance permet d'apprendre ce qui ne peut l'être à l'école. La pédagogie de l'alternance est adaptée à la complexité du métier et aux nouvelles organisations du travail. Dans cette perspective, la pratique devenant objet de réflexion contribue à la création d'un nouveau rapport au savoir et à la construction de l'identité professionnelle. Cette interdépendance façonne les processus d'apprentissage, que ce soit par l'implication quotidienne dans des activités professionnelles ou lors d'exercices organisés intentionnellement sur le lieu de travail. 


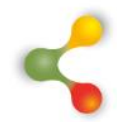

\section{REVUE HYBRIDE DE L'ÉDUCATION}

Pour sa part, Mayen (2018) fait valoir l'importance de la démarche d'analyse du travail pour l'ingénierie du travail apprenant. L'identification au préalable du potentiel d'apprentissage des situations constitue l'ancrage essentiel pour apprendre de et par les situations de travail soutenant ainsi la formation par l'activité et l'expérience. De même, Billett (2011) propose une didactique de la pratique fondée sur l'action et les épistémologies personnelles des acteurs à travers les expériences vécues dans l'environnement de travail.

La dimension du partenariat inclut les aspirations professionnelles des travailleuses et travailleurs (Dignard, 2017), le codéveloppement professionnel (Payette et Champagne, 2000), les environnements de travail (Billett, 2004) ainsi que la qualification et les pédagogies de l'alternance (Geay, 2007).

La modélisation du processus de raisonnement de métier s'inspire des travaux sur la théorie de l'action du philosophe Ricœur (1986). La notion de raison d'agir, en ordonnant la chaine des moyens dans une stratégie, constitue la clé du raisonnement pratique. Cette forme d'intelligence des situations correspond à un processus décisionnel mobilisant l'ensemble des ressources individuelles en vue d'un diagnostic et d'une organisation de stratégies d'action. Cette « délibération » en temps réel comprend des paliers décisionnels et opérationnels (Alexandre, Daigle et Amyot, 2019 ; Alexandre, Thériault et Daigle, 2016).

Cette étude visait deux objectifs opératoires : 1 ) identifier le processus de raisonnement en soudage-montage de personnes enseignantes, formatrices en milieu de travail et travailleuses expérimentées, b) décrire le processus de raisonnement en soudage-montage au regard de l'interdépendance entre la professionnalisation, l'employabilité et le partenariat.

\section{Méthodologie}

Les personnes participantes sont des membres du personnel enseignant $(n=2)$, des travailleuses et des travailleurs $(n=7)$ de la formation professionnelle ayant obtenu un diplôme d'études professionnelles (DEP) ainsi qu'un "compagnon » en entreprise $(n=1)$ en soudage-montage. Le recrutement s'est effectué par contact téléphonique et par la suite en personne auprès des directions d'entreprises en collaboration avec le Comité sectoriel de la main-d'œuvre de la fabrication métallique du Québec et des centres de formation professionnelle. Ce sont des hommes et une femme entre 25 et 70 ans et cumulant au minimum cinq ans de pratique dans le métier $(n=10)^{4}$.

\footnotetext{
${ }^{4}$ Un certificat éthique (CÉR-84-569) a été obtenu du Comité éthique de la recherche de I'UQAR.
} 


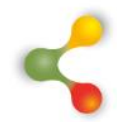

\section{REVUE HYBRIDE DE L'ÉDUCATION}

La première phase de l'étude a consisté à recueillir les différents types de savoir de métier (concepts, procédures et attitudes) des programmes de formation (DEP, ASP et PAMT) en soudage-montage. À la suite de cette analyse documentaire, une proposition théorique constituée d'un groupe d'actions associées à des activités clés a été élaborée. La deuxième phase de l'étude a consisté en trois entretiens non directifs auprès de chacun des participants. Le premier entretien non directif portait sur la validation des résultats de l'analyse documentaire, le second sur l'explicitation des activités clés du processus de raisonnement en soudagemontage et le troisième rendait compte de l'exécution des actions. Afin de procéder à la validation de la proposition théorique du processus de raisonnement en soudage-montage, la chercheure présentait à la personne participante des vignettes sur lesquelles sont inscrites des activités clés et des actions. Tout en verbalisant sa pensée, cette personne associait les vignettes des activités clés à celles des actions incluant l'ajout, le retrait ou la modification d'éléments, assurant ainsi l'explicitation de chacune des activités clés et des actions du processus de raisonnement en soudagemontage. L'exécution commentée et filmée des actions associées à chacune des activités clés préalablement identifiées complétait la deuxième phase de l'étude. Tous les entretiens ont été enregistrés et transcrits verbatim.

Une analyse thématique de contenu (Mucchielli, 2009) a été effectuée. La préparation des données, l'étiquetage des unités de sens, la catégorisation et l'association des catégories et les opérations de synthèse des données ont conduit à l'identification des activités clés et des actions. La démarche d'analyse a mobilisé les modes de raisonnement déductif, inductif ainsi qu'abductif. Notons qu'un mode d'analyse déductive a été mis à contribution par l'élaboration a priori du processus de raisonnement de métier à partir des programmes de formation. Par la suite, en se basant sur les résultats issus des données, des relations ont été générées entre les différents concepts qui ont émergé. Enfin, une zone de tension entre le connu et l'inconnu a permis d'ouvrir sur le concept de l'abduction (Johansson, 2003) qui, tel que mis en place dans cette étude, a complété les modes déductif et inductif permettant de faire ressortir les faits inattendus de l'analyse des données.

\section{Résultats}

La présentation des résultats décrit les actions associées aux activités clés du processus de raisonnement en soudage-montage. En outre, l'analyse des données révèle des interactions entre la professionnalisation, l'employabilité et le partenariat en situation de travail. 


\section{REVUE HYBRIDE DE L'ÉDUCATION}

\subsection{Activités clés et actions du processus de raisonnement en soudage-montage}

Les résultats indiquent la présence de liens entre les activités clés et les actions sous forme de paliers décisionnels. L'exercice d'un processus de raisonnement en soudage-montage assure le déploiement (déroulement) des activités clés et d'un répertoire d'actions en réponse à la complexité de la situation de travail. La figure 2 présentée ci-dessous illustre les actions découlant de chacune des activités clés du processus de raisonnement en soudage-montage.

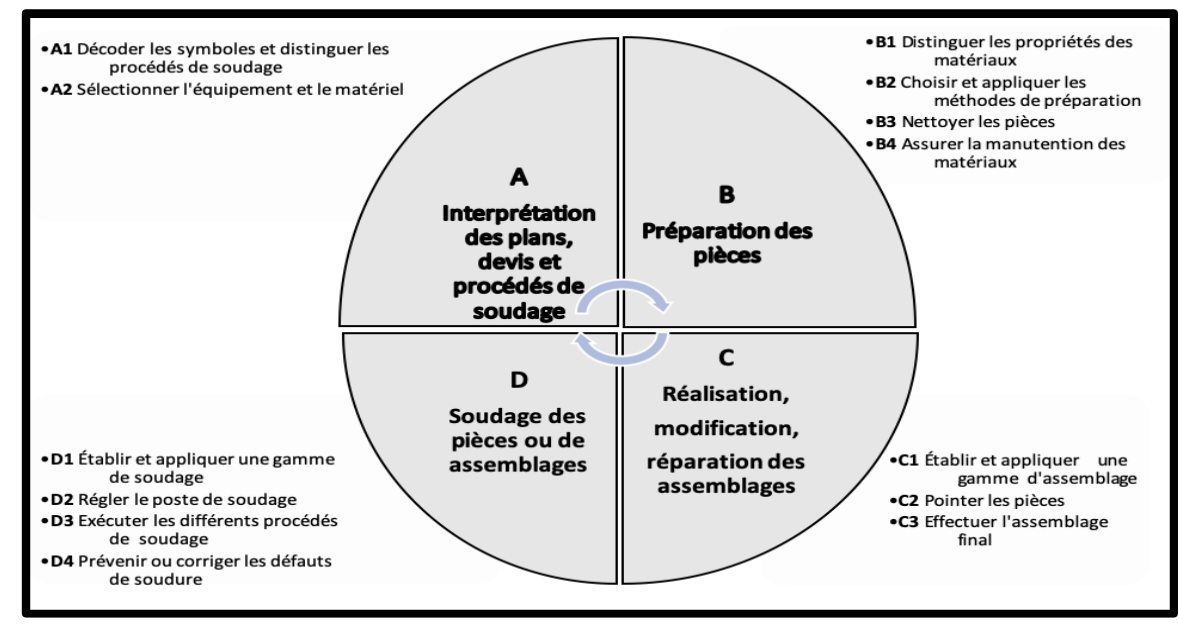

Figure 2 : Activités clés et actions du processus de raisonnement en soudage-montage (Alexandre, Daigle et Amyot, 2019)

Les actions Décoder les symboles et distinguer les procédés de soudage, et Sélectionner l'équipement et le matériel se rapportent à l'activité clé Interprétation des plans, devis et procédés de soudage illustrées dans l'extrait verbatim suivant :

On a toujours besoin d'un plan. Et souvent, des procédures de soudage pour guider l'élève sur les ajustements de la soudeuse en fonction des épaisseurs, des types de joints qu'ils ont à réaliser. Souvent aussi avec la position de soudage qu'ils ont à faire (Paul, enseignant, I.42-45)

La Préparation des pièces intègre les actions suivantes : Distinguer les propriétés des matériaux, Choisir et appliquer les méthodes de préparation, Nettoyer les pièces et Assurer la manutention des matériaux. 


\section{$\&$}

\section{REVUE HYBRIDE DE L'ÉDUCATION}

Quant aux actions Établir et d'appliquer une gamme d'assemblage, Pointer les pièces et Effectuer l'assemblage final, elles s'inscrivent dans l'activité clé Réalisation, modification et réparation des assemblages.

Si c'est une pièce qui a été coupée au chalumeau ou à la torche oxycoupage, on s'assure qu'il n'y ait pas de bavure, que ce soit nettoyé au " grinder »(I.36-38). Ensuite, tu la mets en place [...]. Tu peux la " tacker », [...], l'assembler. Après ça, tu reprends tes mesures avant de souder complètement. ... (Hervé, formateur en milieu de travail, I.43 -45).

Enfin, Établir et appliquer une gamme de soudage, Régler le poste de soudage, Exécuter les différents procédés de soudage et Prévenir ou corriger les défauts de soudure constituent les principales actions de l'activité clé Soudage des pièces ou des assemblages.

De plus, les résultats de l'étude démontrent que chaque action du métier est caractérisée et décrite à partir de repères et d'indicateurs précis, sélectionnés et activés par le professionnel. Ces éléments sont rassemblés sous le terme de paramètres et regroupés en trois grandes familles ou triade. Les paramètres de sécurité sont activés de façon prioritaire lors de l'entrée dans l'action. Les paramètres du métier sous-tendent tout l'exercice des activités clés et des actions du métier. Les paramètres de conformité sont activés lors de chaque contrôle de qualité, et ce, avant de donner le signal du passage à l'étape subséquente.

\subsection{Professionnalisation et processus de raisonnement en soudage- montage}

Les personnes participantes mettent en œuvre des savoirs spécifiques en soudage-montage. L'action Établir et appliquer une gamme de soudage (D1) est le point de départ de l'activité clé Soudage des pièces et des assemblages. Cette action comprend principalement l'étude de la gamme de soudage et l'application des indications de cette gamme sur la ou les pièces à souder incluant la préparation de l'assemblage et du traçage précis des endroits qui seront ultérieurement soudés. Par exemple, lors de l'action Choisir et appliquer les méthodes de préparation (B2) de l'activité clé Préparation des pièces, les paramètres de métier spécifiques aux procédés de coupage sont requis pour ajuster la vitesse de lame et la vitesse de coupe et lors de la procédure de démarrage de l'équipement pour ajuster le jet du liquide de coupe. La vérification qu'aucune pièce n'entre en conflit avec l'opération de coupe est cruciale.

Lorsque ma machine [...] ma scie est ajustée, la bonne vitesse de lame [...] la bonne vitesse de coupe, je peux procéder au démarrage de l'équipement, m'assurer que je n'ai pas de pièce qui entre en conflit avec l'opération de coupe. Par la 


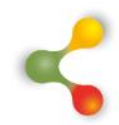

\section{REVUE HYBRIDE DE L'ÉDUCATION}

suite, je peux exécuter la coupe de mon matériel (Paul, enseignant, cap. 11 , secs : 0-60)

Les familles de paramètres permettent de mieux identifier les savoirs professionnels mobilisés dans une situation de travail précise et d'avoir accès à leur configuration à différents moments de la situation. La triade paramétrique met au jour la dynamique interactive des savoirs dans le déroulement d'une action du métier. L'extrait suivant traite de paramètres spécifiques des matériaux.

On a toute la technicalité relative au plan (...) quels types de matériaux devront être utilisés dans l'assemblage, (...) si on parle de G40.21 pour de l'acier. (Carment, I.56-60) c'est de l'acier doux standard. Si on dit 350W, (...) c'est le grade d'acier, W pour «Weldable » pour soudable. Donc, ce sont des types d'acier qu'on va utiliser (Carment, I.62-64).

Le tableau 2 ci-dessous présente les éléments constitutifs de la triade paramétrique en soudage-montage.

\section{Tableau 2 : Triade paramétrique en soudage-montage}

\begin{tabular}{|c|c|c|}
\hline $\begin{array}{l}\text { Paramètres } \\
\text { Sécurité }\end{array}$ & $\begin{array}{l}\text { Paramètres } \\
\text { Métier (assise) }\end{array}$ & $\begin{array}{l}\text { Paramètres } \\
\text { Conformité }\end{array}$ \\
\hline $\begin{array}{l}\text { - Individuelle } \\
\text { - Santé auditive } \\
\text { comportementale } \\
\text { oculaire, respiratoire } \\
\text { - Collective } \\
\text { - Manipulation (produits } \\
\text { chimiques), manutention } \\
\text { (déplacement), } \\
\text { fonctionnement des } \\
\text { outils poste de soudage } \\
\text { /coupage, électricité, } \\
\text { gaz, permis et } \\
\text { procédures }\end{array}$ & $\begin{array}{l}\text { - Matériaux } \\
\text { - Procédés de } \\
\text { coupage, } \\
\text { oxycoupage et } \\
\text { plasma } \\
\text { - Procédés de } \\
\text { soudage } \\
\text { (GMAW/GTAW/TIG) } \\
\text { - Proécédés de } \\
\text { gougeage, (à l'arc- } \\
\text { air)(Gouger signifie } \\
\text { creuser un sillon) }\end{array}$ & $\begin{array}{l}\text { - Matériaux } \\
\text { - Assemblage } \\
\text { (dimensionnelle et } \\
\text { géométrique) } \\
\text { - Gamme de soudage } \\
\text { - Cordon de soudure } \\
\text { - Instruments de } \\
\text { mesure } \\
\text { - Environnement de } \\
\text { travail } \\
\text { - Mesures de sécurité } \\
\text { - Équipements de } \\
\text { protection individuelle } \\
\text { - Postes de travail }\end{array}$ \\
\hline
\end{tabular}

Les résultats présentés dans la section suivante tendent à montrer l'interdépendance des concepts de professionnalisation, d'employabilité et de partenariat lors de l'exercice du processus de raisonnement en soudage-montage. 


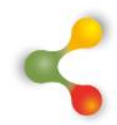

\section{REVUE HYBRIDE DE L'ÉDUCATION}

\subsection{Employabilité et processus de raisonnement en soudage- montage}

\begin{abstract}
L'employabilité se reconnaît à la transférabilité de compétences d'ordre intellectuel, méthodologique, personnel, social et communicationnel (gouvernement du Québec, 2019). Les résultats révèlent l'exercice d'un processus de résolution de problèmes, l'adoption de méthodes de travail efficaces et la collaboration. La prise en compte de la sécurité au travail se traduit par la connaissance et l'activation ciblée des paramètres de sécurité et de conformité laissant présager un impact positif sur l'employabilité. Les paramètres de sécurité en soudage-montage ${ }^{5}$ extraits dans le cadre de cette étude sont des indicateurs de l'application des normes en vigueur dans les milieux investigués. L'extrait verbatim suivant porte sur un paramètre de sécurité individuelle lié à la santé respiratoire.
\end{abstract}

Si c'est un endroit restreint, ce qu'on appelle un espace clos, c'est sûr que ça va nous prendre de la ventilation. Pis avant d'aller dans l'espace clos, ça prend un test d'air avec un permis, comme de quoi l'air n'est pas explosif (Hervé, formateur en milieu de travail /604-606).

Les résultats indiquent l'exercice d'un processus de résolution de problème, par exemple lors de la vérification de la qualité du trait de coupe, des tolérances dimensionnelles et angulaires de la pièce.

La qualité de la coupe va avoir une grande influence sur l'étape qui suit soit l'assemblage du projet. Si les coupes ne sont pas droites, il sera difficile pour le soudeur d'assembler les pièces correctement et faire une soudure conforme au plan [...] respecter les tolérances dimensionnelles [...] On doit entrer dans les tolérances prescrites (Paul, enseignant, cap. 11 , secs : $1: 23-1: 51)$

La collaboration peut s'avérer nécessaire entre autres, lors d'une étape de contrôle de qualité avant de passer à l'étape suivante. L'extrait verbatim ci-dessous concerne les paramètres de conformité du cordon de soudure et de l'assemblage dimensionnel.

II arrive que nous prenions la décision avec le superviseur et le « foreman » (I.595-598). En nettoyant nos morceaux, on voit si nos soudures sont bien faites (I.632-633). Des fois, tu peux regarder le plan et revérifier. Est-ce que j'ai la bonne mesure?

\footnotetext{
5 Les paramètres de sécurité sont régis par les normes et règlements décrétés par l'association canadienne de normalisation (ACNOR). La norme de référence CSA W117.2 établit les règles de sécurité en soudage, coupage et procédés connexes.
} 


\section{$\&$}

\section{REVUE HYBRIDE DE L'ÉDUCATION}

J'ai fini de souder, est-ce trop tordu? (Langis, travailleur, I.635-637)

\subsection{Partenariat et processus de raisonnement en soudage-montage}

Sur le plan du partenariat, la pratique professionnelle contribue à la création d'un nouveau rapport au savoir. L'analyse des actions montre que les personnes participantes font référence aux lieux de travail comme espaces d'apprentissage, et aux interactions entre l'individu, son activité et son environnement. Dans l'extrait verbatim suivant, Carmen, une soudeuse apporte un exemple d'implication quotidienne dans des activités professionnelles.

[...] des gens vont avoir des tâches (...) quand on est dans une microentreprise [...] ce n'est pas rare que des soudeurs fassent les dessins, [...] apportent leur vision [...] le client l'approuve là (Carmen, travailleuse, I.146-150)

\section{Discussion}

La formalisation du processus de raisonnement en soudagemontage met en évidence des manifestations de l'intelligence professionnelle spécifique au métier. L'analyse des données rejoint l'ensemble des auteurs de référence de la recension des écrits à propos du sens de l'action tout en contribuant à le définir. Les résultats correspondent aux travaux menés en didactique professionnelle concernant l'interprétation de l'action. En outre, les résultats accordent une large place à l'activation des paramètres de sécurité, de métier et de conformité. L'examen de la triade paramétrique dans différentes situations de travail a permis de préciser l'organisation cognitive de l'action.

Wittorski (2016) milite pour une meilleure compréhension des rapports réciproques des environnements, des organisations, des groupes et des individus porteurs de logiques différentes. Ces résultats rejoignent les propos de Vincens (2005), pour qui les acteurs doivent eux-mêmes donner sens à l'adéquation entre la formation et l'emploi. Le Bureau international du travail (2011) insiste sur la nécessité d'établir de solides ponts entre l'enseignement professionnel, le développement des compétences et le monde du travail. Cet arrimage essentiel vise l'acquisition par les travailleuses et les travailleurs des compétences exigées par l'évolution des demandes du marché du travail.

La mise au jour des activités clés et des actions du métier va dans le sens des travaux d'Holgado et Balleux (2015) quant à la compréhension des milieux dans lesquels se forment les professionnels. Mayen (2018) soutient que l'on doit s'écarter du travail pour mieux l'apprendre. En considérant le travail et la formation comme des contextes favorisant les apprentissages, le processus de raisonnement en soudage-montage porté 


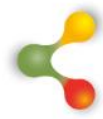

\section{REVUE HYBRIDE DE L'ÉDUCATION}

par ces résultats s'apparente à une métacompétence spécifique du métier. Les travaux de Correa Molinea et al. (2010) définissent la métacompétence comme une compétence de second niveau qui soutient le développement de toutes les autres, qu'elles soient disciplinaires et transversales. Ceci laisse présager que la réflexion sur les activités clés et les actions du processus de raisonnement en soudage-montage pourrait devenir un levier de professionnalisation, d'employabilité et de partenariat tel que mis de l'avant dans la modélisation du développement des compétences durables en formation professionnelle et technique. Toutefois, Mayen (2018) prévient qu'une analyse de l'expérience d'apprentissage de travail réussie requiert la rencontre de conditions et de compétences.

Sur le plan de la professionnalisation, les activités clés et les actions du processus de raisonnement de métier en soudage-montage configurent spécifiquement l'interdépendance des savoirs détenus par les différents acteurs à partir d'activités réelles du domaine dans le sens émis par Holgado et Mayen (2017) ainsi que par Leplay (2006). Plus particulièrement, Champy-Remoussenard (2008) souligne que la professionnalisation est un indicateur de la relation entre l'éducation, la formation et l'emploi et fait valoir la nécessité de rendre lisibles les compétences. Les résultats de l'étude vont dans le même sens et facilitent l'élaboration et le partage d'une définition commune de l'agir professionnel. Le cadre conceptuel du développement des compétences durables inscrit les interactions entre l'individu, son activité et son environnement dans un rapport spécifique à l'activité sociale. L'analyse de l'activation paramétrique en soudage-montage précise une forme de répertoire qui témoigne de l'organisation de chacune des actions du métier, tout en donnant des informations détaillées en continu sur l'environnement immédiat dans lequel l'action prend forme et se développe.

Ces résultats appuient également les travaux de Fray et Picouleau (2010) concernant l'usage de pratiques, de vocabulaire et de gestes communs associés au développement de l'identité professionnelle. Par exemple, les paramètres du métier illustrés dans le tableau 2 font notamment référence à l'ensemble des procédés tels que l'oxycoupage, le coupage plasma, le soudage GMAW, GTAW et TIG, etc.

Sous l'angle de l'employabilité, les résultats indiquent une mobilisation de compétences transversales d'ordre intellectuel et méthodologique. C'est le cas de l'activité clé d'Interprétation des plans, devis et procédés de soudage qui s'appuie entre autres sur la capacité à résoudre des problèmes et à exploiter l'information. La capacité à se donner des méthodes de travail efficaces est présente lors de la Réalisation, modification et réparation des assemblages ainsi que lors du Soudage des pièces et de l'assemblage. L'accès au processus de raisonnement de métier facilite la «lisibilité » et la caractérisation des compétences requises. Ces résultats corroborent l'ensemble des travaux présentés dans la recension des écrits concernant l'ancrage de 


\section{$\&$}

\section{REVUE HYBRIDE DE L'ÉDUCATION}

compétences d'employabilité en contexte disciplinaire. En revanche, comme les personnes participantes possédaient cinq années d'expérience en soudage-montage, les points critiques du parcours de développement n'ont pu être repérés. En conséquence, les résultats actuels ne permettent pas d'établir de distinction entre les experts et les novices.

Bien que peu d'éléments ressortent de l'examen des données sur le plan du partenariat, l'extrait suivant du verbatim du formateur en entreprise illustre le décalage entre la formation et l'emploi dans l'apprentissage du travail :

[...] je leur avais dit que j'étais capable de former les gars. Je suis capable de leur montrer à souder. Parce que quand ils arrivent de l'école, un jeune qui sort de l'école, il ne sait pas souder, ce n'est pas vrai. II ne sait pas souder (I.398-400) [...] à l'école, il est tout le temps bien placé, la petite plaque, il la met comme il veut, il la positionne comme il veut (Hervé, formateur en milieu de travail, I.412-413).

II s'agit, dans le sens émis par Dignard (2017), d'une mesure subjective de l'adéquation entre la formation et l'emploi fondée sur le jugement d'une personne. Ce constat d'un formateur pourrait se rapprocher des propos de Mayen (2018) sur l'importance de la rencontre de conditions favorables sur le potentiel d'apprentissage des situations de travail. Néanmoins, il semble souhaitable que la modélisation de l'agir professionnel puisse servir d'outil réflexif autant en éducation qu'en milieu de travail.

L'apprentissage du travail ne peut faire l'impasse sur l'interdépendance entre la professionnalisation, l'employabilité et le partenariat. La modélisation des activités clés et des actions déployées lors du processus de raisonnement en soudage-montage constitue un levier de développement des compétences durables en formation professionnelle et technique. 


\section{REVUE HYBRIDE DE L'ÉDUCATION}

\section{Références}

Action Canada (2013). Conjuguer l'éducation au futur. Adapter les systèmes éducatifs canadiens pour le 21 ième siècle. http://www.actioncanada.ca/fr/project/future-tense-adaptingcanadian-education-systems-21st-century/

Alexandre, M. (2018). Paramètres associés aux activités clés du processus de raisonnement de métier : Repenser l'agir professionnel en soudagemontage. [communication orale]. 86 ${ }^{\mathrm{e}}$ congrès de l'ACFAS. Chicoutimi, Qc, Canada.

Alexandre, M., Daigle, G. et Amyot, D. (2019). L'agir professionnel et le processus de raisonnement de métier. Éducation Permanente, 219(Hors-série AFPA), 159-167.

Alexandre, M., Thériault, N. et Daigle G. (2016). Modélisation du savoir professionnel du travailleur, du compagnon et de l'enseignant en techniques d'usinage : le processus de raisonnement de métier. Revue Travail et Apprentissages, 18(2), 7-23.

Barbier, J.M. (2012). Tendances d'évolution de la formation des adultes. Dans M. Bailleul, J.-F. Themines et R. Wittorski (dir.), Expériences et développement professionnel des enseignants: formation, travail, itinéraire professionnel (p.11-25). Octares.

Bernier, A., Michaud, R. et Poulet, N. (2017). L'adéquation entre les compétences et l'emploi occupé : pratiques des employeurs dans les $P M E$ québécoises du secteur manufacturier. Rapport de recherche. TELUQ : Université du Québec.

Billett, S. (2011). Learning in the circumstances of work: the didactics of practice. Éducation et didactique, 5(2), 125-146.

Billett, S. (2004). Workplace participatory practices: Conceptualizing workplaces as learning environments. Journal of Workplace Learning, 16(6), 312-324.

Bricler, M. (2009). Des compétences d'employabilité durable pour sécuriser les parcours professionnels des individus. Projectique, 3(3), 95-101.

Bros, F., Aït-Abdesselam, N. et Bellgarde, K. (2019). À qui profite la compétence transversale?, Éducation Permanente, 219(Hors-série AFPA), 139-148. 


\section{REVUE HYBRIDE DE L'ÉDUCATION}

Bureau international du travail (2011). Une main-d'œuvre qualifiée pour une croissance forte, durable et équilibrée. Une stratégie de formation du G20.

Champy-Remoussenard, P. (2008). Incontournable professionnalisation. Savoirs, 2(17), 51-61. DOI 10.3917/savo.017.0051

Conference Board du Canada (2019). Compétences relatives à l'employabilité. $\quad$ https://www.conferenceboard.ca/docs/defaultsource/educ-public/esp2000f.pdf?sfvrsn=b3c7a141 0

Correa Molina, E., Collin, S., Chaubet, P. et Gervais, C. (2010). Concept de réflexion : un regard critique. Éducation et francophonie, 38(2), 135154. https://doi.org/10.7202/1002160ar

Dignard, H. (2017). L'adéquation formation-emploi : une notion polysémique. Repéré à URL http://www.icea.qc.ca/site/fr/actualites/l'adéquation-formation-emploiune-notion-polysémique

Doray, P. B., Simoneau, F. et Solar-Pelletier, L. (2017). L'adéquation entre la formation et l'emploi : le cas de la formation sur mesure dans l'enseignement supérieur à Montréal. Montréal: CIRST, CIRDEF, UQAM. $\quad$ https://www.cirdef.uqam.ca/wpcontent/uploads/2017/07/PSRA-Rapport-Adequation-Final-26042017$\underline{\text { VF.pdf }}$

Dujardin, J.M. (2013). Compétences durables et transférables. Clés pour l'employabilité. De Boeck Supérieur.

Emploi-Québec. (2018). Soudeurs/soudeuses et opérateurs/opératrices de machines à souder et à braser (CNP 7237). http://imt.emploiquebec.gouv.qc.ca/mtg/inter/noncache/contenu/asp/mt g122 descrprofession 01.asp?lang=FRAN\&pro $=7237$

Fournier, C., Lambert, M. et Marion-Vernoux, I. (2017). Le travail au cœur des apprentissages en entreprise. Bulletin de Recherches EmploiFormation. CÉREQ, Bref, (353), 1-4.

Fray, A. et Picouleau, S. (2010). Le diagnostic de l'identité professionnelle : une dimension essentielle pour la qualité au travail. Management et Avenir, 38(8), 72-88.

Geay, A. (2007). L'alternance comme processus de professionnalisation : implications didactiques. Éducation Permanente, 172, 27-38. 


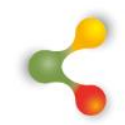

\section{REVUE HYBRIDE DE L'ÉDUCATION}

Gouvernement du Canada (2017). Bâtir une classe moyenne forte. Budget 2017. URLhttps://www.budget.gc.ca/2017/docs/plan/budget-2017-

fr.pdf

Gouvernement du Canada (2018). Emploi-Avenir Québec. https://www.guichetemplois.gc.ca/pieces jointesfra.do?cid=10238\&lang=fra\#stats

Gouvernement du Québec (2013). Tous pour l'emploi. Québec : Une impulsion nouvelle avec les partenaires. Ministère du Travail, de l'Emploi et de la Solidarité sociale. http://laboratoiredesmetiers.com/wp-content/uploads/2015/06/guidedidactique-machiniste.pdf

Gouvernement du Québec. (2019). Exploration de la formation professionnelle. Ministère de l'éducation et de l'enseignement supérieur.

http://www.education.gouv.qc.ca/fileadmin/site web/documents/educa tion/jeunes/pfeq/PFEQ exploration-formation-professionnelle.pdf

Holgado, O. et Balleux, A. (2015). Explorer la relation formation-travailapprentissage à partir de différents cadres théoriques. Regards croisés sur la formation professionnelle [communication orale]. $83^{e}$ congrès de l'ACFAS. Rimouski, Qc, Canada.

Holgado, O. et Mayen, P. (2017). Former les formateurs de l'enseignement professionnel à l'analyse du travail en didactique professionnelle, Revue Travail et Apprentissages, 17, 17-35.

Jobert, G. (2002). La professionnalisation entre compétences et reconnaissance sociale. Dans L. Paquay et P. Perrenoud (dir.), Formateurs d'enseignants, quelle professionnalisation? (p.247-260). De Boeck.

Johansson, R. (2003). Case study methodology [communication orale].Conférence internationale "Methodologies in Housing Research », Royal Institute of Technology et International Association of People-Environment Studies, Stockholm.

Leplay, E. (2006). Co-construction de savoirs professionnels par la recherche: vers un dispositif expérimental en formation initiale de travailleurs sociaux. Esprit critique, 8(1), 1-19.

Mayen, P. (2018). S'écarter du travail pour mieux l'apprendre. Une réflexion pour l'ingénierie de formation en situation de travail et pour la conception d'organisations apprenantes. Éducation Permanente, 216(3),141-157. 


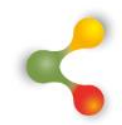

\section{REVUE HYBRIDE DE L'ÉDUCATION}

McGuinness, S. Pouliakas et Redmond, P. (2017). How Useful is the Concept of Skills Mismatch?, International labour organization. Suisse : Genève. $\quad$ https://www.ilo.org/wcmsp5/groups/public/---ed emp/--ifp skills/documents/publication/wcms 552798.pdf

Mucchielli, A., (2009). Thématique (analyse de contenu). Dictionnaire des méthodes qualitatives en sciences humaines (3e éd.). Armand Colin.

Nendaz, M., Charlin, B., Leblanc, V. et Bordage, G. (2005). Le raisonnement clinique : données issues de la recherche et implications pour l'enseignement. Pédagogie médicale,6(4), 235-254.

Organisation internationale du travail (2014). World of work Report .2014 Developing with jobs. Executive summary. Research Department.

Pastré, P. (2002). L'analyse du travail en didactique professionnelle. Revue Française de Pédagogie, 138, 9-17.

Pastré, P., Mayen, P. et Vergnaud, G. (2006). La didactique professionnelle. Revue française de pédagogie, 154, 145-198.

Payette A. et Champagne C. (2000). Le Groupe de co-développement professionnel. Presses de l'Université du Québec.

Perform (2012). La fabrication métallique au Québec. Comité sectoriel de la main-d'œuvre dans la fabrication métallique industrielle.

Perform (2014). Sommaire du diagnostic sectoriel de la fabrication métallique industrielle au Québec 2014-2016. Comité sectoriel de la main-d'œuvre dans la fabrication métallique industrielle. Commission des partenaires du marché du travail du Québec.

Perform (2015). Comité sectoriel de la main-d'œuvre dans la fabrication métallique industrielle. Soudeurs. http://www.comiteperform.ca/Soudeurs-

Perform (2017). Formez pour performer : Le métier de machiniste. Comité sectoriel de la main-d'œuvre dans la fabrication métallique industrielle. Québec: gouvernement du Québec. http://www.comiteperform.ca/Machinistes-

Perrenoud, P. (2004). Adosser la pratique réflexive aux sciences sociales de l'éducation. Dans J.- F. Inisan (dir.), Analyse de pratiques et attitude réflexive en formation (p. 11-32). Reims.

Rasul, M. S., Rauf, A. R. et Mansor, A. N. (2013). Employability Skills Indicator as Perceived by Manufacturing Employers. Asian Social Science, 9(8), 42-46. 


\section{REVUE HYBRIDE DE L'ÉDUCATION}

Ricoeur, P. (1986). Du texte à l'action. Essais d'herméneutique II. Seuil.

Rogalski, J. (2004). La didactique professionnelle : une alternative aux approches de « cognition située » et « cognitiviste » en psychologie des acquisitions. Activités, 1(2), 103-120. http://activites.revues.org/1259.

Santelmann, P. (2019). Partir des compétences tranversales pour lire autrement le travail. Éducation Permamente, 219(Hors-série AFPA), 711.

Simoneau, F. (2017). Adéquation formation-emploi : de quoi parle-t-on?, Bulletin de l'Observatoire compétences-emploi, 1(8). http://www.oce.uqam.ca/article/adequation-formation-emploi-de-quoiparle-t-on/

Trilling, B. et Fadel, C. (2009). 21st Century Skills: Learning for Life in Our Times. Jossey-Bass.

Trottier, C. (2005). L'analyse des relations entre le système éducatif et le monde du travail en sociologie de l'éducation : vers une recomposition du champ d'études?, Education et sociétés, 2(16), 77-97. DOI 10.3917/es.016.0077.

Vincens, J. (2005). L'adéquation formation-emploi. Dans J.-F. Giret, A. Lopez et J. Rose (dir.), Des formations pour quels emplois? (p.149162). La Découverte, CÉREQ.

Voogt, J. et Pareja Roblin, N. (2012). A comparative analysis of international framework for $21^{\text {st }}$ century competences: Implications for national curriculum policies. Journal of Curriculum Studies, 3(44), 299321.http://dx.doi.org/10.1080/00220272.2012.668938

Wanlin, P. (2009). La pensée des enseignants lors de la planification de leur enseignement. Revue française de pédagogie,166, 89-128. http://rfp.revues.org/1294

Wittorski, R. (2016). La professionnalisation en formation: textes fondamentaux. Presses universitaires de Rouen et du Havre. 